\title{
A Novel Feature Selection Method Based on Binary Differential Evolution and Feature Subset Correlation for Microarray Data
}

\author{
Weidong Xie ( $\sim$ 1910638@stu.neu.edu.cn ) \\ Northeastern University https://orcid.org/0000-0003-1930-4509 \\ Yuhuan Chi \\ Northeastern University \\ Linjie Wang \\ Northeastern University \\ Kun Yu \\ Northeastern University \\ Wei Li \\ Northeastern University
}

\section{Research Article}

Keywords: Differential evolution, Feature selection, Relevance, Biomarkers

Posted Date: February 7th, 2022

DOI: https://doi.org/10.21203/rs.3.rs-1283185/v1

License: (c) (i) This work is licensed under a Creative Commons Attribution 4.0 International License. Read Full License 
Highlights

\section{A Novel Feature Selection Method Based on Binary Differential Evolution and Feature Subset Correlation for Microarray Data}

Weidong Xie, Yuhuan Chi,Linjie Wang,Kun Yu,Wei Li.

- A binary quantization method of differential evolution algorithm for microarray data is proposed, which effectively balances the global search performance and convergence speed of the algorithm.

- The scaling factor of the differential evolution algorithm is improved, and an adaptive approach is used to ensure the population diversity and the global search ability of the population in the early stage of the algorithm.

- The feature subset coefficients are added to the fitness function of the algorithm to ensure that the requested feature subset has the minimum redundancy and enhance the effectiveness of the feature subset. 


\title{
A Novel Feature Selection Method Based on Binary Differential Evolution and Feature Subset Correlation for Microarray Data ${ }^{\star}$
}

\author{
Weidong $\mathrm{Xie}^{a}$, Yuhuan $\mathrm{Chi}^{a}$, Linjie Wang ${ }^{a}$, Kun $\mathrm{Yu}^{b}$ and Wei Li.,* \\ ${ }^{a}$ School of Computer Science and Engineering, Northeastern University, China \\ ${ }^{b}$ Biomedical and Information Engineering School, Northeastern University, China \\ ${ }^{c}$ Key Laboratory of Intelligent Computing in Medical Image (MIIC), Northeastern University, Ministry of Education, China
}

\section{ARTICLE INFO}

\section{Keywords:}

Differential evolution

Feature selection

Relevance

Biomarkers

\begin{abstract}
A B S T R ACT
Obtaining diagnostically important features from microarray data can help researchers understand diseases and study drugs. The challenges come from the high dimensionality, small sample size of the data and the high redundancy among features. To solve this problem, we propose a new hybrid feature selection method based on differential evolution algorithm. The proposed method the proposed method adopts a new binary quantization method to adapt to the characteristics of highdimensional data, and improves the scaling factor and mutation operator to balance the global search performance and convergence speed of the algorithm. The proposed method also proposes the concept of feature subset correlation in the fitness function to ensure the low redundancy of feature subsets. The experimental results show that the proposed method is advanced and the selected features, are with low redundancy and can effectively distinguish between positive and negative samples.
\end{abstract}

\section{Introduction}

With advances in biotechnology, researchers have easy access to large scale, diverse and high-dimensional genomics and proteomics data. Although the increase in data size brings new possibilities for analyzing causative genes of complex diseases and conducting drug studies, etc., however, analyzing such data is challenging due to the redundant information contained in the data. For example, human microarray data contains a large amount of genetic information. However, only a few genes are associated with Leukemia, and these genes associated with specific diseases are also called biomarkers [1]. Understanding how to obtain disease-specific biomarkers from microarray data is important for clinical diagnosis, drug development and disease understanding.

In order to discover biomarkers from microarray data, researchers use feature selection mothods to deal with the microarray data [2]. Depending on the evaluation metrics, feature selection can be classified as Filter, Wrapper and Embedded [3]. Filter has the highest efficiency, based on data attributes and statistical analysis to quickly filter features that are irrelevant or weakly correlated with the label, however, it is difficult to guarantee the classification accuracy, common filter methods are ReliefF [4], T-test [5], Chi-squared test [6], and Maximal Information Coefficient (MIC) [7], Gini Index [8], Kullback-Leibler divergence [9], Fisher score [10], Laplace operator score [11]. Wrapper methods usually use classification accuracy as the evaluation index and use heuristic search algorithm to traverse the feature space, so the wrapper methods have the lowest efficiency and

*Corresponding author

@1910638@stu.neu.edu.cn (W. Xie); 2071743@stu.neu.edu.cn (Y. Chi); 1971660@stu.neu.edu.cn (L. Wang); yukun@bmie.neu. edu.cn (K. Yu); liwei@cse.neu.edu.cn (W. Li.)

ORCID(s): 0000-0003-1930-4509 (W. Xie) highest accuracy, and common Wrapper methods are Recursive Feature Elimination (RFE) [12], Genetic Algorithm (GA) [13], K-Nearest Neighbor (KNN) [14] and Particle Swarm Optimization (PSO) [15]. Embedded methods rely on specific machine learning models to select features by constructing feature weight coefficients generated by the model, which is a good balance between algorithmic efficiency and accuracy. common Embedded methods are Supported Vector Machine Recursive Feature Elimination (SVM-RFE), Decision Tree (DT) [16], Random Forest Algorithm (RF) [17], and Lasso Regression (LR) [18].

In order to obtain better efficiency and classification accuracy, researchers usually combine methods such as Filter and Wrapper to form hybrid feature selection methods, in order to optimize the feature selection process. There is a large amount of literature on hybrid feature selection methods [19, 20, 21, 22, 23, 24, 25]. However, they mostly assume that features are independent of each other. However, in microarray data, it has been shown that there is a significant interaction relationship between biomarkers [26].

Based on the above problems, this paper proposes a feature selection algorithm based on differential evolutionary algorithm applied to microarray data. The method first uses a T-test to initially filter redundant features, and then uses a binary approach to model the feature selection problem, and proposes a novel binary quantization method and an adaptive scaling factor to balance the global search performance and convergence speed of the algorithm. In addition, to ensure the obtained feature subsets have the minimum redundancy, the proposed method redesign the fitness function by including the average correlation of the feature subsets as part of the fitness function. 


\section{Related wrok}

For the feature selection task of microarray data, existing research are dominated by hybrid feature selection methods. lu et al. [27] designed a feature selection method based on mutual information (MI) and adaptive genetic algorithm (AGA) for microarray data. The method first uses mutual information as a filtering method to achieve the initial filtering of features. However, the mutual information stage does not take into account the continuity of feature attributes. The adaptive genetic algorithm is then used to perform further feature selection and finally obtain a subset of features.

Salem et al. [20] pointed out that although no one feature selection technique can significantly outperform other feature selection methods, hybrid methods can usually achieve superior performance. He proposed a hybrid feature selection method combining Information Gain (IG) and genetic algorithm, where IG is used as a filtering method for initial feature screening, followed by genetic algorithm for further feature selection, and finally Genetic Programming (GP) for classification.

Theera et al. [28] designed a neuro-fuzzy based Firefly algorithm for feature selection of microarray data. The method does not have a clear distinction between Filter and Wrapper, but embeds the neuro-fuzzy technique into the merit-seeking process and the evaluation process of the firefly algorithm. Results from multiple datasets demonstrate the advancement and effectiveness of the method.

Wang et al. [29] proposed a hybrid feature selection method in which an innovative Markov Blank (MB) decision technique was used as a filtering method to reduce the time overhead of the Wrapper method while effectively removing redundant features. The sequential forward selection method (SFS) is then used as a wrapper method to perform further feature selection. The experimental results show that this method can effectively improve the efficiency of the algorithm and reduce the redundant features.

Aziz et al. [30] proposed a hybrid feature selection method based on Independent Component Analysis (ICA) and Artificial Intelligence Bee Colony (ABC), which uses Nave Bayes (NB) as a classifier and optimizes the feature vectors in ICA by $A B C$ algorithm to achieve feature selection, and proved the effectiveness of the method by ablation experiments and comparison experiments with advanced algorithms.

Moradi et al. [31] proposed a method called HPSO-LS uses a local search strategy which is embedded in the particle swarm optimization to select the less correlated and salient feature subsetThe method uses an embedded particle swarm optimization approach to optimize the local search strategy for selecting features with low and significant correlation. The proposed method compares multiple Filter methods including information gain, term variance, fisher score and mRMR and five well-known wrapper-based methods including genetic algorithm, particle swarm optimization, simulated annealing and ant colony optimization, demonstrating the effectiveness of the methods.
Table 1

Strategies used in existing hybrid feature selection methods

\begin{tabular}{cccc}
\hline \multirow{2}{*}{ Author } & \multicolumn{3}{c}{ Stage } \\
\cline { 2 - 4 } & Filter & Wrapper & Combine \\
\hline Lu et al.[19] & MI & AGA & Not applied \\
Salem et al.[20] & IG & GA & Not applied \\
Wang et al. [29] & MB & SFS & Not applied \\
Aziz et al. [30] & ICA & ABC & Not applied \\
Theera et al. [28] & Not applied & Firefly & Neuro-fuzzy \\
Moradi et al. [31] & Not applied & HPSO & LS \\
Vanitha et al.[32] & MI & Not applied & SVM(LOOCV) \\
\hline
\end{tabular}

Vanitha et al. [32] proposed a feature selection method based on Mutual information and SVM, which uses MI to identify the information between features and labels, uses SVM as a classifier, and employs Leave-one-out CrossValidation (LOOCV) as a cross-validation method to test the method's validity. The experimental results show that the method can select the most significant subset of features.

Table 1 summarizes the main strategies of the hybrid approach, combine indicates that some strategy is embedded in the Wrapper approach. The combination of these methods achieves better classification accuracy and convergence speed. In our proposed method, T-test is used as the filter methods, differential evolution is used as the Wrapper method, and the feature subset redundancy is combined as the fitness function. The main innovations of this paper are as follows.

- A binary quantization method of differential evolution algorithm for microarray data is proposed, which effectively balances the global search performance and convergence speed of the algorithm.

- The scaling factor of the differential evolution algorithm is improved, and an adaptive approach is used to ensure the population diversity and the global search ability of the population in the early stage of the algorithm.

- The feature subset correlation coefficients are added to the fitness function of the algorithm to ensure that the requested feature subset has the minimum redundancy and enhance the effectiveness of the feature subset.

The rest of the paper is organized as follows: section 2 presents the related work, section 3 describes the hybrid feature selection algorithm proposed in this paper, subsection 4 describes the experimental part, and finally subsection 5 concludes the whole paper.

\section{The proposed feature selection method}

This section describes the proposed feature selection processing flow, firstly, how to pre-process the data and the initial filtering of features using T-test, followed by our improved binary difference evolution algorithm flow. Finally we present the improvement strategies for the scaling 
factor and fitness function of the binary difference evolution algorithm.

\subsection{Datasets Pre-processing}

For the overall sample data, we first treated all missing values and outliers as missing values and used the KNN method to fill in the missing values. To ensure the efficiency of the filtering method, we performed independent sample $\mathrm{t}$-test on the overall sample. The independent sample t-test can be used to determine the statistical differences between subjects after different treatments, and is very suitable for preprocessing microarray data. The statistic $t$ for the independent sample t-test is calculated as shown in Equation 1.

$$
t=\frac{\bar{x}-\bar{y}}{S_{w} \sqrt{\frac{1}{m}+\frac{1}{n}}} \sim t(m+n-2)
$$

where $x$ and $y$ represent the mean value of two samples between positive and negative samples, $m$ and $n$ represent the size of two samples, $S_{w}$ is calculated as shown in Equation 2.

$$
S_{w}=\frac{1}{m+n+1}\left[(m-1) S_{1}^{2}+(n-1) S_{2}^{2}\right]
$$

where $S_{1}$ and $S_{2}$ subscales represent the variance of the two samples. Usually we retain 500 sets of features for the next step of feature selection based on the test statistic.

\subsection{Binary differential evolution algorithm}

Differential evolution (DE) is a simple and effective global optimization algorithm. However, the standard DE and most of its variants operate in continuous space and cannot directly solve the optimization problem of binary coding. Here we present the flow of the binary differential evolution algorithm.

In the binary differential evolution algorithm, we first perform population initialization, using $N_{p}$ binary vectors of dimension $D$ to represent each population, with each individual represented as shown in Equation 3.

$$
\boldsymbol{x}_{i, G}\left(i=1,2, \cdots, N_{\mathrm{P}}\right)
$$

where $i$ denotes the sequence of individuals in the population, $G$ denotes the number of iterations of the algorithm, $N_{p}$ denotes the population size, and $N_{p}$ remains constant throughout the optimization process.

In order to establish the initial points for optimal search, the population must be initialized. Usually the initialization of the DE algorithm is chosen randomly from a constrained range of given boundary values. For the feature selection task of microarray data, we define the vector length of an individual as the feature dimension, and each vector indicates whether the feature is selected or not. That is, for a sample with 5 features, we initialize $p=[0,0,0,1,1]$ to indicate that the last two features are selected.
The key point of the differential evolution algorithm is calculating the variance vector. The variance vector $H_{i}(g)$ calculation of the traditional binary differential evolution algorithm evolved through the differential evolution algorithm, as shown in Equation 4, where three individuals $X_{p 1}$, $X_{p 2}$, and $X_{p 3}$ are randomly selected from the population and $i \neq p 1 \neq p 2 \neq p 3$. This type of direct string manipulation does not effectively mimic the continuous DE algorithm. It performs poorly on high-dimensional data [33].

$$
H_{i}(g)=X_{p 1}(g)+F \cdot\left(X_{p 2}(g)-X_{p 3}(g)\right)
$$

Therefore, in the improved binary difference evolution algorithm, we use the vector $u_{i}(g)$ represents the $j$-th binary code of the final mutation vector, which is shown as Epuation 5.

$$
u_{i}^{j}(g)=\left\{\begin{array}{c}
1, \text { if } p r \geq \operatorname{rand}(0,1) \text { and } X_{p 3}^{j}(g)=1 \\
0, \text { otherwise }
\end{array}\right.
$$

where rand $(0,1)$ indicates a random value between 0 and 1 , $p r$ is calculated as shown in Equation 6.

$$
p r=\frac{e^{\operatorname{diff}_{i}^{j}(g)}-e^{-\operatorname{diff}_{i}^{j}(g)}}{e^{\operatorname{diff}_{i}^{j}(g)}+e^{-\operatorname{diff}_{i}^{j}(g)}}
$$

The $\operatorname{diff}_{i}^{j}(g)$ represents the $j$-th dimension binary code of difference vector, which is calculated in Equation 7.

$$
\operatorname{diff}_{i}^{j}(g)=\left\{\begin{array}{l}
0, \text { if } X_{p 1}^{j}(g)=X_{p 2}^{j}(g) \\
F X_{i}^{j}(g), \text { otherwise }
\end{array}\right.
$$

where $F$ is the scaling factor, which is shown in Equation 8. In the next subsection, we will focus on explaining the improvements to $F$.

$$
F=\left\{\begin{array}{l}
\operatorname{rand}[0,0.5],(g / G) \geq 0.5 \\
\operatorname{rand}[0.5,1],(g / G)<0.5
\end{array}\right.
$$

The crossover process can ensure the diversity of the population. The improved adaptive crossover operator is calculated as shown in Equation 9, where $\alpha$ is a parameter, we have tested that 0.9 is a suitable value for microarray data. The final selection operator is computed as shown in Equation 10. In our feature selection process, the fitness function is quantified using classification accuracy and feature subset correlation, which will be discussed in detail later.

$$
\begin{gathered}
C R=\alpha \frac{2 e^{-(g / G)}}{e^{(g / G)}+e^{-(g / G)}} \\
x_{i}(g+1)= \begin{cases}v_{i}(g) & , \text { if } f\left(v_{i}(g)\right) \text { better than } f\left(x_{i}(g)\right) \\
x_{i}(g) & , \text { otherwise }\end{cases}
\end{gathered}
$$

where $x_{i}(g+1)$ is a new individual, $f(*)$ is the fitness function. 


\subsection{Adaptive scaling factor}

In the above binary DE algorithm, the calculation of $F$ (Equation 8) is fixedly constrained to two ranges. Too large of $F$ in the DE algorithm will lead to low efficiency of the algorithm search and low accuracy of the global optimal solution obtained, while too small will reduce the population diversity and tend to converge prematurely. Therefore, we propose an adaptive scaling factor $F$ that can be dynamically adjusted according to the progress of the algorithm search, which is calculated as shown in Equation 11

$$
F=F_{0} \times 2^{\lambda}
$$

where $F_{0}$ denotes the variance operator, usually taken as 0.5 , $\lambda$ is calculated in Equation 12.

$$
\lambda=e^{1-\frac{G_{\mathrm{m}}}{G_{\mathrm{m}}+1-G}}
$$

where $G_{\mathrm{m}}$ denotes the maximum number of iterations and $\mathrm{G}$ denotes the current number of iterations. Therefore, the adaptive variation operator at the beginning of the algorithm is $2 F_{0}$, which has a large value and can ensure individual diversity at the beginning of the algorithm, and as the algorithm proceeds, the variation operator gradually decreases and will be infinitely close to $F_{0}$ at a later stage to retain good information, avoid the optimal solution from being destroyed, and increase the probability of searching for the global optimal solution.

\subsection{Feature correlation-based fitness function}

The calculation of fitness in the standard DE algorithm and some heuristic algorithms usually uses the classification accuracy of a given classifier. In order to avoid incorporating too many redundant features in the feature subset, we improve the calculation of the fitness function of the DE algorithm by using the feature subset correlation coefficient as an evaluation metric together with the classification accuracy to supervise the evolutionary direction of the algorithm. First, we define the feature subset correlation coefficient corr, which is calculated as the average of the sum of the inter-correlation coefficients of all features in that feature subset. Thus the improved fitness Fitness is calculated as shown in Equation 13.

$$
\text { Fitness }=\operatorname{rand}(0.6,1) * a c c+(1-\operatorname{rand}(0.6,1) * c o r r))
$$

where acc is the mean value of the five-fold cross-validated classification accuracy for a given classifier, SVM was used as the classifier in the experiments, and Pearson correlation coefficient was used to evaluate the feature similarity. Since the classification accuracy has the most important impact on the results, we assigned more weights. We also added random perturbations to both evaluation metrics to ensure the randomness of the search process.
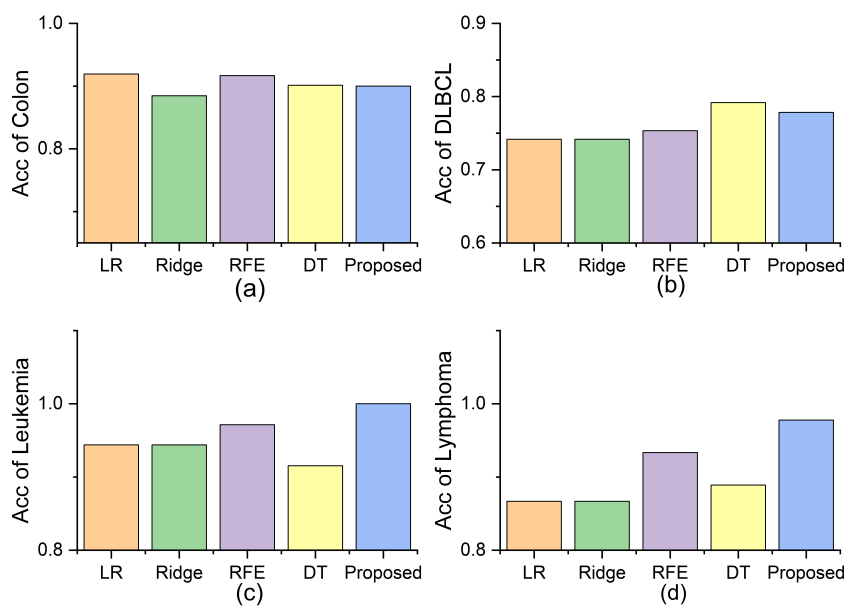

Figure 1: Comparison of the classical feature selection method and the proposed method, Figure (a) shows the results for the Colon dataset, Figure (b) for the DLBCL dataset, Figure (c) for the Leukemia dataset, and Figure (d) for the Lymphoma dataset. SVM five-fold cross-validation was used as the classification accuracy, and the number of features for all methods remained consistent with the proposed method.

\section{Experiment}

\subsection{Datasets and parameters}

Four DNA microarray datasets were used in this paper, namely Colon, Leukemia, Lymphoma and DLBCL, the details of these datasets are shown in Table 2. Colon is a colon cancer dataset containing 40 tumor samples and 22 normal samples containing 2000 genetic information. Leukemia is a leukemia dataset containing 25 samples of Acute Myeloid Leukemia (AML) and 47 samples of Acute Lymphocytic Leukemia (ALL); each sample contains 7129 genes. Lymphoma is a dataset containing 22 tumor samples and 23 normal samples; each sample contains 4026 genetic information. DLBCL is a lymphoma data set, including 59 samples of diffuse large B-cell lymphoma (DLBCL) and 19 samples of follicular lymphoma (FL). Each sample includes 7070 gene information.

This experiment uses Python 3.9 as the development environment. In the experiment, T-test is used for the preliminary filtering of features, and 500 features are retained and input into the improved BDE algorithm. All the parameter settings of our algorithm are shown in Table 3.

\subsection{Comparing with classical feature selection methods}

In this section, we compare the proposed method with the classical feature selection methods, which include linear regression (LR), ridge regression (Ridge), recursive feature elimination (RFE), and decision tree (DT), and we select the same number of features as the proposed method, use SVM as the classification model, and the average of the five-fold cross-validation as the final classification accuracy, and the detailed results are shown in Table 4 and Figure 1. 
Table 2

Description of the datasets used in this paper.

\begin{tabular}{ccccccc}
\hline Data & Features & Samples & Pos & Neg & Ur & Description \\
\hline Colon & 2000 & 62 & 40 & 22 & 1.82 & tumour (40) and normal (22) \\
Leukemia & 7129 & 72 & 47 & 25 & 1.88 & ALL (47) and AML (25) \\
Lymphoma & 4026 & 45 & 22 & 23 & 0.95 & germinalcentre (22) and activated B-like DLBCL (23) \\
DLBCL & 7070 & 77 & 58 & 19 & 3.05 & Diffuse Large B-Cell Lymphoma(59) and Follicular Lymphoma(19) \\
\hline
\end{tabular}

Table 3

Proposed algorithm parameters

\begin{tabular}{lll}
\hline Parameters & Values & Description \\
\hline$N_{P}$ & 20 & Population size \\
$G$ & 500 & Number of iterations \\
$F$ & Equation 11 & Scaling factor \\
$C R$ & 0.5 & Crossover probability \\
$P$ & 500 & Chromosome number \\
$\alpha$ & 0.9 & Adaptive crossover factor \\
\hline
\end{tabular}

The results in Table 4 and Figure 1 show that on the Colon dataset the proposed method does not differ much from the classical methods, on the DLBCL dataset the proposed method outperforms LR, Ridge and RFE, and the DT method outperforms the proposed method. However, on the Leukemia and Lymphoma datasets, the proposed method outperforms all classical feature selection methods by a wide margin. It should be noted that the proposed method also ensures that the subset of features has minimal correlation, which increases the clinical diagnostic significance of the selected features.

\subsection{Comparing with advanced feature selection methods}

This section compares the proposed method with some published advanced hybrid feature selection methods on four datasets, and the detailed results are shown in Table 5. It can be seen that on different datasets, the proposed method can significantly reduce the number of features, and the method proposed in all comparison methods can obtain the most streamlined subset of features, which is important to reduce the cost of clinical diagnosis. In addition, the proposed method achieves the highest classification accuracy with few features, so the proposed method is advanced.

\subsection{Biomarker Analysis}

In this section, we will further analyze the subset of features selected by the proposed method, namely biomarkers. Figure 2 shows the distribution of the selected features in positive and negative samples on different datasets, each color represents a feature. It can be seen that the distribution of selected features in positive and negative samples varies greatly in different datasets, that is, these features can distinguish positive and negative samples very well and have significance.

Taking the Leukemia dataset as an example, we also retrieved PubMed regarding the relationship between Leukemia and our selected features, first we converted the

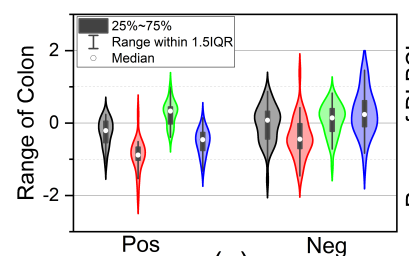

(a)

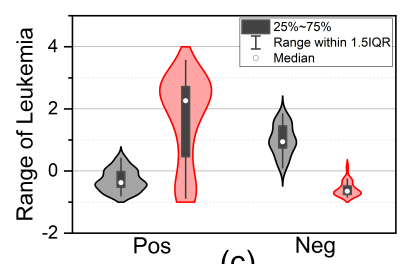

(c)

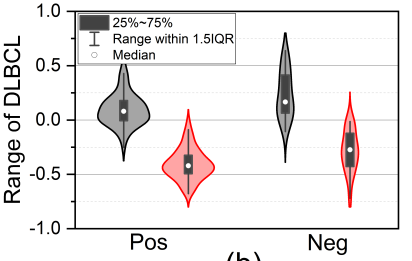

(b)

(d)

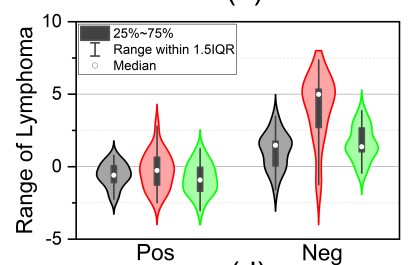

Figure 2: Distribution of selected features in positive and negative samples of different datasets. Figure $(a)$ is the Colon dataset, Figure (b) is the DLBCL dataset, Figure (c) is the Leukemia dataset, and Figure (d) is the Lymphoma dataset. Each color in the graph represents a feature, and the features of the same color show the difference in the distribution of positive and negative samples.

corresponding probe ID to gene ID and used the gene ID as the retrieved value. Probe M23197_at corresponds to gene CD33, which was reported to be associated with Leukemia in 2024 publications on PubMed. Probe D88270_at corresponds to the gene VPREB1, and there are 16 publications on PubMed reporting VPREB1 to be associated with Leukemia. We also obtained the interaction between the two genes from GeneMINIA, as shown in Figure 3. It can be seen that the two genes we selected do not have a significant interaction relationship with low redundancy.

\section{Conclusion}

In this paper, a novel feature selection method is proposed, which uses t-test as a filter method to initially filter features and to reduce the time overhead of the Wrapper algorithm. Subsequently, an improved binary differential evolution algorithm is used to further select features. For microarray data, the proposed method improve the variation operator and scaling factor of the differential evolution algorithm, and the concept of subset correlation coefficient is proposed and added to the fitness function to guide the search process of the algorithm. Experimental results show 
Table 4

Comparison of the classical feature selection method and the proposed method

\begin{tabular}{ccccccccc}
\hline \multirow{2}{*}{ Methods } & \multicolumn{2}{c}{ Colon } & \multicolumn{2}{c}{ DLBCL } & \multicolumn{2}{c}{ Leukemia } & \multicolumn{2}{c}{ Lymphoma } \\
\cline { 2 - 9 } & Features & Acc & Features & Acc & Features & Acc & Features & Acc \\
\hline LR & 4 & 0.9192 & 2 & 0.7417 & 2 & 0.9438 & 3 & 0.8667 \\
Ridge & 4 & 0.8846 & 2 & 0.7417 & 2 & 0.9438 & 3 & 0.8667 \\
RFE & 4 & 0.9167 & 2 & 0.7533 & 2 & 0.9714 & 3 & 0.9333 \\
DT & 4 & 0.9013 & 2 & 0.7917 & 2 & 0.9152 & 3 & 0.8889 \\
Proposed & 4 & 0.9000 & 2 & 0.7783 & 2 & 1.0000 & 3 & 0.9777 \\
\hline
\end{tabular}

\section{Table 5}

The proposed method is compared with the advanced hybrid feature selection method. Filter represents the Filter method used in the method, Wrapper represents the Wrapper method used in the method, and Combin represents the embedded method used in the Wrapper method.

\begin{tabular}{clcllll}
\hline Datasets & Literature & Features & Acc & Filter & Wrapper & Combine \\
\hline Colon & Lu et al.[19] & 202 & 0.8341 & MI & AGA & Not applied \\
& Salem et al.[20] & 60 & 0.8548 & IG & GA & Not applied \\
& Proposed & 4 & 0.9000 & T-test & DE & Corr \\
DLBCL & Theera et al. [28] & 13 & 0.8381 & Not applied & FA & Neuro-fuzzy \\
& Wang et al. [29] & 15 & 0.8090 & MB & SFS & Not applied \\
& Proposed & 2 & 0.7783 & T-test & DE & Corr \\
Leukemia & Aziz et al. [30] & 12 & 0.9868 & ICA & ABC & Not applied \\
& Wang et al. [29] & 8.3 & 0.9610 & MB & SFS & Not applied \\
& Proposed & 2 & 1.0000 & T-test & DE & Corr \\
Lymphoma & Moradi et al. [31] & 50 & 0.8771 & Not applied & HPSO & LS \\
& Vanitha et al.[32] & 4 & 0.9090 & MI & Not applied & SVM(LOOCV) \\
& Proposed & 3 & 0.9777 & T-test & DE & Corr \\
\hline
\end{tabular}

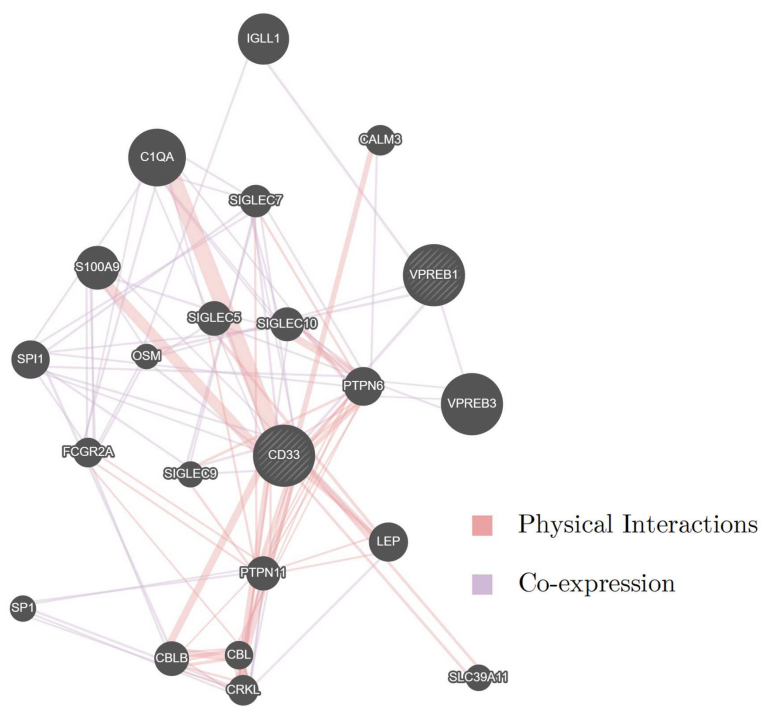

Figure 3: From the characteristic interaction network obtained from GeneMANIA, it can be seen that the selected genes of the proposed method have no direct interaction and low redundancy.

that the proposed method can effectively reduce the number of features, ensure the classification accuracy, and select a subset of features with low redundancy.

\section{Conflict of interest}

The authors declare that they have no conflict of interest.

\section{Ethical approval}

This study was performed using available datasets, as per my compliance with ethical standards there were no human or animal participants, and therefore, the study did not require ethics approval.

\section{Informed consent}

All authors were aware of the publication of the paper and agreed to its publication

\section{Funding}

This document is the results of the research project funded by National key research and development program, China (2021YFC2701003), the Fundamental Research Funds for the Central Universities (N2016006).

\section{References}

[1] Abdul Wahid, Dost Muhammad Khan, Sajjad Ahmad Khan, Ijaz Hussain, and Zardad Khan. Robust regularization for highdimensional cox's regression model using weighted likelihood criterion. Chemometrics and Intelligent Laboratory Systems, 213:104285, 2021. 
[2] Nicoletta Dessì and Barbara Pes. Similarity of feature selection methods: An empirical study across data intensive classification tasks. Expert Systems with Applications, 42(10):4632-4642, 2015.

[3] J. C. Ang, A. Mirzal, H. Haron, and Hna Hamed. Supervised, unsupervised, and semi-supervised feature selection: A review on gene selection. IEEE/ACM Transactions on Computational Biology Bioinformatics, 13(5):971-989, 2016.

[4] Igor Kononenko, Edvard Šimec, and Marko Robnik-Šikonja. Overcoming the myopia of inductive learning algorithms with relieff. Applied Intelligence, 7(1):39-55, 1997.

[5] Nina Zhou and Lipo Wang. A modified t-test feature selection method and its application on the hapmap genotype data. Genomics, proteomics \& bioinformatics, 5(3-4):242-249, 2007.

[6] Huan Liu and Rudy Setiono. Chi2: Feature selection and discretization of numeric attributes. In Proceedings of 7th IEEE International Conference on Tools with Artificial Intelligence, pages 388-391. IEEE, 1995.

[7] Chen Lin, T Miller, D Dligach, RM Plenge, EW Karlson, and G Savova. Maximal information coefficient for feature selection for clinical document classification. In ICML Workshop on Machine Learning for Clinical Data. Edingburgh, UK, 2012.

[8] Laura Elena Raileanu and Kilian Stoffel. Theoretical comparison between the gini index and information gain criteria. Annals of Mathematics and Artificial Intelligence, 41(1):77-93, 2004.

[9] Mark Hall and Lloyd Smith. Practical feature subset selection for machine learning. Proceedings of the 21st Australasian Computer Science Conference, 98, 011996.

[10] Quanquan Gu, Zhenhui Li, and Jiawei Han. Generalized fisher score for feature selection. arXiv preprint arXiv:1202.3725, 2012.

[11] Xiaofei He, Deng Cai, and Partha Niyogi. Laplacian score for feature selection. volume 18, 012005.

[12] Ke Yan and David Zhang. Feature selection and analysis on correlated gas sensor data with recursive feature elimination. Sensors and Actuators B: Chemical, 212:353-363, 2015.

[13] Xiang Li, Ningchuan Xiao, Christophe Claramunt, and Hui Lin. Initialization strategies to enhancing the performance of genetic algorithms for the p-median problem. Computers \& Industrial Engineering, 61(4):1024-1034, 2011.

[14] Subhajit Kar, Kaushik Das Sharma, and Madhubanti Maitra. Gene selection from microarray gene expression data for classification of cancer subgroups employing pso and adaptive k-nearest neighborhood technique. Expert Systems with Applications, 42(1):612-627, 2015.

[15] Ioan Cristian Trelea. The particle swarm optimization algorithm: convergence analysis and parameter selection. Information processing letters, 85(6):317-325, 2003.

[16] Gary Stein, Bing Chen, Annie S Wu, and Kien A Hua. Decision tree classifier for network intrusion detection with ga-based feature selection. In Proceedings of the 43rd annual Southeast regional conference-Volume 2, pages 136-141, 2005.

[17] Kun-Huang Chen, Kung-Jeng Wang, Min-Lung Tsai, Kung-Min Wang, Angelia Melani Adrian, Wei-Chung Cheng, Tzu-Sen Yang, Nai-Chia Teng, Kuo-Pin Tan, and Ku-Shang Chang. Gene selection for cancer identification: a decision tree model empowered by particle swarm optimization algorithm. BMC bioinformatics, 15(1):49, 2014.

[18] Valeria Fonti and Eduard Belitser. Feature selection using lasso. $V U$ Amsterdam Research Paper in Business Analytics, 30:1-25, 2017.

[19] Huijuan Lu, Junying Chen, Ke Yan, Qun Jin, Yu Xue, and Zhigang Gao. A hybrid feature selection algorithm for gene expression data classification. Neurocomputing, 256:56-62, 2017.

[20] Hanaa Salem, Gamal Attiya, and Nawal El-Fishawy. Classification of human cancer diseases by gene expression profiles. Applied Soft Computing, 50:124-134, 2017.

[21] Hala Alshamlan, Ghada Badr, and Yousef Alohali. mrmr-abc: a hybrid gene selection algorithm for cancer classification using microarray gene expression profiling. Biomed research international, 2015,2015
[22] Indu Jain, Vinod Kumar Jain, and Renu Jain. Correlation feature selection based improved-binary particle swarm optimization for gene selection and cancer classification. Applied Soft Computing, 62:203215, 2018.

[23] Parham Moradi and Mozhgan Gholampour. A hybrid particle swarm optimization for feature subset selection by integrating a novel local search strategy. Applied Soft Computing, 43:117-130, 2016.

[24] Salam Salameh Shreem, Salwani Abdullah, and Mohd Zakree Ahmad Nazri. Hybrid feature selection algorithm using symmetrical uncertainty and a harmony search algorithm. International Journal of Systems Science, 47(6):1312-1329, 2016.

[25] Kun Yu Weidong Xie, Linjie Wang and Wei Li. Ilrc: a hybrid biomarker discovery algorithm based on improved 11 regularization and clustering in microarray data. BMC Bioinformatics, 22(1):514, 2021.

[26] A Jl, B Iyc, and C Chj. An efficient multivariate feature ranking method for gene selection in high-dimensional microarray data. Expert Systems with Applications, 166, 2020.

[27] Ling Wang, Xiping Fu, Yunfei Mao, Muhammad Ilyas Menhas, and Minrui Fei. A novel modified binary differential evolution algorithm and its applications. Neurocomputing, 98:55-75, 2012. Bio-inspired computing and applications (LSMS-ICSEE' 2010).

[28] P. Jinthanasatian, S. Auephanwiriyakul, and N. Theera-Umpon. Microarray data classification using neuro-fuzzy classifier with firefly algorithm. In 2017 IEEE Symposium Series on Computational Intelligence (SSCI), 2018.

[29] A. Wang, N. An, J. Yang, G. Chen, L. Li, and G. Alterovitz. Wrapperbased gene selection with markov blanket. Computers in Biology Medicine, 81(Complete):11-23, 2017.

[30] R. Aziz, C. K Verma, and N. Srivastava. A novel approach for dimension reduction of microarray. Computational Biology Chemistry, 71:161-169, 2017.

[31] H. Lu, J. Chen, K. Yan, Q. Jin, Y. Xue, and Z. Gao. A hybrid feature selection algorithm for gene expression data classification. Neurocomputing, 256(sep.20):56-62, 2016.

[32] Cda Vanitha, D. Devaraj, and M. Venkatesulu. Gene expression data classification using support vector machine and mutual informationbased gene selection. Procedia Computer Science, 47:13-21, 2015.

[33] Yu Chen, Weicheng Xie, and Xiufen Zou. A binary differential evolution algorithm learning from explored solutions. Neurocomputing, 149:1038-1047, 2015. 